\title{
A HIERARCHY BASED ON A TYPE TWO OBJECT
}

\author{
BY

\section{J. R. SHOENFIELD $\left({ }^{1}\right)$}

The object of hierarchy theory is to break down certain classes of predicates into subclasses of gradually increasing complexity. An excellent example is furnished by the hyperarithmetical hierarchy. Here each class in the hierarchy is obtained from the previous class by adding a single number quantifier. The Souslin-Kleene theorem [2, XXIV] then tells us that the predicates occurring in the hierarchy are just the $\Delta_{1}^{1}$ predicates.

A direct generalization of this result to the next higher type presents difficulties. In fact, it appears from [6] that a hierarchy in which a single type one quantifier is added at each stage could not exhaust the $\Delta_{1}^{2}$ predicates. However, results on higher type objects suggest a different generalization. Kleene [3, XLVIII] has shown that the $\Delta_{1}^{1}$ predicates are just the predicates recursive in the type two object $E$ defined by

$$
\begin{aligned}
E(\alpha) & =0 & & \text { if } \operatorname{Et}(\alpha(t)=0), \\
& =1 & & \text { otherwise. }
\end{aligned}
$$

Thus the predicates occurring in the hyperarithmetical hierarchy are just those which are recursive in $E$.

We shall generalize this by producing for each type two object $\left({ }^{2}\right) F$ in which $E$ is recursive a hierarchy consisting of the predicates recursive in $F$. This hierarchy is quite similar to the hyperarithmetical hierarchy $\left({ }^{3}\right)$. The major difference is that instead of defining the ordinal notations prior to the hierarchy, we use the predicates in the hierarchy to construct new ordinal notations. That this is necessary for extensions of the hyperarithmetical hierarchy is well known (see [3, p. 140]).

The restriction that $E$ be recursive in $F$ seems to be necessary because there is no suitable jump operation associated with a type two object in which $E$ is not recursive. In any case, this does not seem a very significant restriction. The restriction to type two objects is much more severe. The results given here certainly do not extend in a straightforward manner to the next higher type. Whether or not there

Received by the editors June 5, 1967.

(1) This research was supported by the National Science Foundation.

$\left({ }^{2}\right)$ For the case in which $F$ is the object $E_{1}$ defined by Tugue, a different hierarchy has been discovered by Richter [5].

$\left({ }^{3}\right)$ In presenting our hierarchy, we have followed Kleene's presentation of the hyperarithmetical hierarchy quite closely. Our work could be simplified a little by following some of the more recent presentations of the hyperarithmetical hierarchy. 
are fundamental difficulties in constructing hierarchies based on type three objects remains to be discovered.

We now suppose that a type two object $F$ in which $E$ is recursive is fixed. We define the jump operation $j$ associated with $F$ as follows: if $A$ is a set (of numbers), then $j(A)$ is the set of numbers $x$ such that $\left\{(x)_{0}\right\}^{A}$ is a total function $\phi$ and $F(\phi)$ $=(x)_{1}$. (As usual, $\{e\}^{A}$ is the partial function which is recursive in $A$ with Gödel number $e$.) The ordinary jump of $A$, designated by oj( $A)$, is recursive in $j(A)$. For we have

$$
x \in o j(A) \leftrightarrow\{x\}^{A}(x) \text { is defined. }
$$

We can find a $y$ depending recursively on $x$ such that

$$
\{y\}^{A}(z) \simeq 0 \cdot\{x\}^{A}(x)
$$

for all $z$. Then if $a$ is the value of $F$ at the zero function, we have $x \in o j(A) \leftrightarrow$ $\langle y, a\rangle \in j(A)$.

We write $A \leqq_{e} B$ to mean that $A$ is recursive in $B$ with Gödel number $e$. It is easy to verify that there is a recursive function $K$ such that

$$
A \leqq_{e} B \rightarrow j(A) \leqq_{K(e)} j(B) .
$$

The above argument shows that there is a number $q$ such that

$$
o j(A) \leqq_{q} j(A)
$$

for all $A$. This implies that there is a number $r$ such that

$$
A \leqq r j(A)
$$

for all $A$.

We shall now define a set $O$ of ordinal notations; a relation $<_{0}$ among ordinal notations; and, for each $a \in O$, an ordinal $|a|$ and a set $H_{a}$. (All of these should carry a superscript $F$ to indicate their dependence on $F$; but we omit this because $F$ is fixed.) The (inductive) definition has three clauses.

(O1) $1 \in O ; \neg(x<01) ;|1|=0 ; H_{1}(x) \leftrightarrow x=x$.

(O2) If $a \in O$, then $2^{a} \in O ; x<_{0} 2^{a}=\left(x<_{0} a \vee x=a\right) ;\left|2^{a}\right|=|a|+1 ; H_{2^{a}}=j\left(H_{a}\right)$.

(O3) Suppose that $a \in \in^{\circ} O$; that $\{e\}^{H_{a}}$ is a total function $\phi$; that $\phi(n) \in O$ and $\phi(n)$ $<_{\circ} \phi(n+1)$ for all $n$; and that $\phi(0)=a$. Then $3^{a} 5^{e} \in O ; x<_{0} 3^{a} 5^{e} \leftrightarrow E n\left(x<_{0} \phi(n)\right)$; $\left|3^{a} 5^{e}\right|=\sup _{n}|\phi(n)|$; and $H_{3^{a} 5^{e}}(x) \leftrightarrow H_{\phi\left((x)_{0}\right)}\left((x)_{1}\right)$.

We shall write $a^{*}$ for $2^{a}$. If $b=3^{a} 5^{e}$, then $\{e\}^{H_{a}}$ is designated by $\phi_{b}$.

The basic properties of $O$ can be proved in the usual way (see [1]). In particular, if $a \in O$, then the set of $b$ such that $b<_{0} a$ is well-ordered by $<_{0}$, and the ordinal of this well-ordering is $|a|$.

UNIQUENESS THEOREM. There is a partial recursive function $L$ such that if $a, b \in O$ and $|a| \leqq|b|$, then $L(a, b)$ is defined and $H_{a} \leqq_{L(a, b)} H_{b}$. 
Proof. Our proof is modeled after Spector's proof [7] in the ordinary case. Along with $L$, we define a partial recursive function $M$ such that if $b \in O$, then $M(b)$ is defined and, for $y \in 0$,

$$
\{M(b)\}^{H_{b^{*}}}(y)=0 \leftrightarrow|y|<|b| .
$$

We define $L$ and $M$ simultaneously by means of the recursion theorem and prove that they have the desired properties by induction on $|b|$. We may thus suppose that $L\left(a^{\prime}, b^{\prime}\right)$ and $M\left(b^{\prime}\right)$ are defined for $\left|a^{\prime}\right| \leqq\left|b^{\prime}\right|<|b|$. We must show how to decide if $H_{a}(x)$ from $H_{b}$, and, for $y \in O$, how to decide if $|y|<|b|$ from $H_{b^{*}}$.

Case 1: $b=1$. Then $a=1$; so $H_{a}(x)$ is true and $|y|<|b|$ is false.

Case 2: $b=b^{*}$. If $a=a^{*}$, then $H_{a^{\prime}}<_{L\left(a^{\prime}, b^{\prime}\right)} H_{b^{\prime}}$ by induction hypothesis; so we may take $L(a, b)=K\left(L\left(a^{\prime}, b^{\prime}\right)\right)$ by (1). If $a$ is not of the form $a^{*}$, then $|a|<|b|$; so $|a| \leqq\left|b^{\prime}\right|$. Thus $H_{a} \leqq_{L\left(a, b^{\prime}\right)} H_{b^{\prime}}$ and $H_{b^{\prime}} \leqq_{r} H_{b}$. We can use these to compute a Gödel number of $H_{a}$ from $H_{b}$.

Now let $y \in O$. If $y=1$, then $|y|<|b|$. If $y=y^{*}$, then

$$
|y|<|b| \leftrightarrow\left|y^{\prime}\right|<\left|b^{\prime}\right| .
$$

Thus we can tell if $|y|<|b|$ from $H_{b}$ by induction hypothesis and hence from $H_{b^{*}}$ by (3). Now let $y=3^{x} 5^{e}$. Since $x=\phi_{y}(0)<_{0} y,|y|<|b|$ implies $|x|<|y| \leqq\left|b^{\prime}\right|$. We therefore first test to see if $|x|<\left|b^{\prime}\right|$; this can be done from $H_{b}$ and hence from $H_{b^{*} \text {. }}$ Assume that $|x|<\left|b^{\prime}\right|$. Then

$$
|y|<|b| \leftrightarrow(n)\left(\left|\phi_{y}(n)\right|<\left|b^{\prime}\right|\right) .
$$

For $n$ fixed, $\phi_{y}(n)=\{e\}^{H_{x}}(n)$ can be calculated from $H_{b}$ by using $L\left(x, b^{\prime}\right)$ and $r$; and we can then decide if $\left|\phi_{y}(n)\right|<\left|b^{\prime}\right|$ by using $M\left(b^{\prime}\right)$. It follows that we can decide if $|y|<|b|$ from $o j\left(H_{b}\right)$, and hence, by (2), from $H_{b^{*}}$.

Case 3: $b=3^{c} 5^{e}$. First assume that $|a|<|b|$. Now

$$
H_{\phi_{b}(n)}(z) \leftrightarrow H_{b}(\langle n, z\rangle) ;
$$

so $H_{\phi_{b}(\pi)}$ is recursive in $H_{b}$ uniformly in $n$. Taking $n=0$ and recalling that $\phi_{b}(0)=c$ and $\phi_{b}=\{e\}^{H_{c}}$, we see that $\phi_{b}$ is recursive in $H_{b}$. Now $|a|<\left|\phi_{b}(n)\right|$ for some $n$. Given $n$, we can calculate $\phi_{b}(n)$ and $\phi_{b}(n+1)$ from $H_{b}$. We can then tell if $|a|<\left|\phi_{b}(n)\right|$ from $H_{d}$, where $d=\phi_{b}(n)^{*}$. But $|d| \leqq\left|\phi_{b}(n+1)\right|<|b|$; so we can decide if $|a|<\left|\phi_{b}(n)\right|$ from $H_{\phi_{b}(n+1)}$ and hence from $H_{b}$. Thus using $H_{b}$, we can actually find an $n$ such that $|a|<\left|\phi_{b}(n)\right|$. Since

$$
H_{a} \leqq_{L\left(a, \phi_{b}(n)\right)} H_{\phi_{b}(n)}
$$

we can decide if $H_{a}(x)$ from $H_{\phi_{b}(n)}$ and hence from $H_{b}$.

Now assume only that $|a| \leqq|b|$. If $a$ is not of the form $3^{d} 5^{f}$, then $|a|<|b|$, and we can proceed as above. Suppose that $a=3^{d} 5^{f}$. Then $H_{a}(x) \leftrightarrow H_{j}\left((x)_{1}\right)$ with $j=$ $\phi_{a}\left((x)_{0}\right)$. Moreover, $|j|<|a| \leqq|b|$. Hence we can decide if $H_{a}(x)$ by the above if we 
can compute $j$ from $H_{b}$. Now $|d|<|a| \leqq|b|$; so by the above, we can find a Gödel number of $H_{d}$ from $H_{b}$. Since $\phi_{a}=\{f\}^{H_{d}}$, we can compute $j=\phi_{a}\left((x)_{0}\right)$ from $H_{b}$.

Now let $y \in O$. Then $|y|<|b| \leftrightarrow E n\left(|y|<\left|\phi_{b}(n)\right|\right)$. Given $n$, we can calculate $\phi_{b}(n)$ and decide if $|y|<\left|\phi_{b}(n)\right|$ from $H_{b}$ as described above. Hence we can decide if $|y|<|b|$ from $o j\left(H_{b}\right)$, and hence, by (2), from $H_{b}$.. This completes the proof.

We shall need an addition function $t_{o}$. This is a partial recursive function defined by the recursion theorem so as to satisfy

$$
a+o 1 \simeq a, \quad a+o b^{*} \simeq(a+o b)^{*}, \quad a+{ }_{o} 3^{b} 5^{e} \simeq 3^{a+o b} 5^{f},
$$

where $f$ is computed from $a, b, e$, and an index of $t_{o}$ as follows. We first compute an index $g$ such that $\{g\}^{H_{b}}(n) \simeq a+o\{e\}^{H_{b}}(n)$; and we then choose $f$ by the uniqueness theorem so that

$$
\{f\}^{H_{a+o b}(n)} \simeq\{g\}^{H_{b}}(n)
$$

(under the assumption that $|b| \leqq\left|a+_{o} b\right|$ ). The only properties of this function which we shall need are

$$
\begin{aligned}
& \left|a+{ }_{o} b\right|=|a|+|b| \\
& b \neq 1 \rightarrow a<_{o} a+{ }_{o} b
\end{aligned}
$$

for $a, b \in O$.

Let $\alpha$ be a total function recursive in $H_{a}$ such that $\alpha(n) \in O$ for all $n$. Then there is a $b \in O$ such that $|\alpha(n)|<|b|$ for all $n$. To see this, choose $f$ so that

$$
\{f\}^{H_{a}}(0)=a, \quad\{f\}^{H_{a}}(n+1)=\{f\}^{H_{a}}(n)+_{o} \alpha(n)^{*}
$$

and let $b=3^{a} 5^{f}$. The required properties of $b$ then follow from (4) and (5). It is clear that $b$ may be computed from $a$ and a Gödel number of $\alpha$ from $H_{a}$.

Principal Theorem. A predicate of number variables is recursive in $F$ if and only if it is recursive in $H_{a}$ for some $a \in O$.

Proof. To prove the if part, we define a partial recursive function $Z$ such that if $a \in O$, then $Z(a)$ is defined and is an index of $H_{a}$ from $F$. The definition is by the recursion theorem, and the proof is by induction on $|a|$. The least trivial case is when $a=a^{\prime *}$. To obtain $Z(a)$ from $Z\left(a^{\prime}\right)$, it suffices to show that $H_{a}=j\left(H_{a^{\prime}}\right)$ is recursive in $H_{a^{\prime}}$ and $F$ uniformly in $H_{a^{\prime}}$. By definition, $j(A)$ is recursive in $A, F$, and $E$ uniformly in $A$; since $E$ is recursive in $F$, the desired result follows.

For the converse, we define partial recursive functions $G$ and $P$ and prove

$$
\{e\}^{F}[x]=P\left(H_{G(e, x)}^{\prime}, e, x\right)
$$

whenever the left side is defined. (Here the square bracket notation is from [3], and $H_{a}^{\prime}$ is the representing function of $H_{a}$.) To see that this suffices, assume that $\{e\}^{F}$ is total. Then by the above, there is a $b \in O$ such that $|G(e, x)| \leqq|b|$ for all $x$. It then follows from (6) and the uniqueness theorem that $\{e\}^{F}$ is recursive in $H_{b}$. 
It is well known that the scheme (S5) of [3] may be omitted, at the cost of adding a scheme introducing a few particular functions of type zero arguments. To simplify the proof, we suppose this done.

We define $P$ and $G$ by the recursion theorem, and prove (6) by induction on $\{e\}^{F}[x]$. We must define $G(e, x)$, show that it is in $O$, and show how to compute $\{e\}^{F}[x]$ from $H_{G(e, x)}, e$, and $x$. Suppose that $(e)_{0}=i$, so that $\{e\}^{F}$ is defined under (Si). If $i=1,2,3$, or 5 , then $\{e\}^{F}$ is a recursive function independent of $F$; so we may take $G(e, x)=1$. If $i=9,\{e\}^{F}(x)=\left\{e^{\prime}\right\}^{F}\left(x^{\prime}\right)$, where $e^{\prime}$ and $x^{\prime}$ may be calculated from $e$ and $x$. We may then take $G(e, x)=G\left(e^{\prime}, x^{\prime}\right)$. A similar treatment holds for $i=6$. The cases $i=7$ and $i=8$ cannot occur with type one arguments. Hence only $i=0$ and $i=4$ remain.

If $i=0,\{e\}^{F}[x]=F\left(\lambda t\left\{e^{\prime}\right\}^{F}\left[x_{t}^{\prime}\right]\right)$ where $e^{\prime}$ and $x_{t}^{\prime}$ can be computed from $e, x$, and $t$. For each $t, G\left(e^{\prime}, x_{t}^{\prime}\right)$ is defined and in $O$. Hence, using an index of $G$, we can compute a $b \in O$ such that $\left|G\left(e^{\prime}, x_{t}^{\prime}\right)\right|<|b|$ for all $t$. Using an index of $P$ and the uniqueness theorem, we get an index of $\lambda t\left\{e^{\prime}\right\}^{F}\left[x_{t}^{\prime}\right]$ from $H_{b}$. We can therefore compute $\{e\}^{F}[x]$ from $j\left(H_{b}\right)$; so we may take $G(e, x)=b^{*}$.

If $i=4$, then

$$
\{e\}^{F}[x]=\left\{e^{\prime}\right\}^{F}\left[H\left(\left\{e^{\prime \prime}\right\}^{F}[x], x\right)\right]
$$

where $e^{\prime}$ and $e^{\prime \prime}$ can be computed from $e$ and $H$ is a recursive function. Set

$$
a=G\left(e^{\prime \prime}, x\right), \quad y=H\left(\left\{e^{m}\right\}^{F}[x], x\right), \quad b=G\left(e^{\prime}, y\right) .
$$

We propose to set $G(e, x)=c$, where $c \in O,|a| \leqq|c|$, and $|b| \leqq|c|$. Assume that this is done. Given $e$ and $x$, we compute $\{e\}^{F}[x]$ from $H_{c}$ as follows. We first compute $b$ and $c$. By the induction hypothesis and the uniqueness theorem, we can compute $\left\{e^{\prime \prime}\right\}^{F}[x]$ from $H_{c}$. We then compute $y$ and $b$. Using the induction hypothesis and the uniqueness theorem again, we can compute $\{e\}^{F}[x]=\left\{e^{\prime}\right\}^{F}[y]$ from $H_{c}$.

It remains to compute such a $c$ from $e$ and $x$. We first compute $a$. By induction hypothesis, $\left\{e^{\prime \prime}\right\}^{F}[x]$ can be computed from $H_{a}$; so $y$ and $b$ can be computed from $H_{a}$. It follows that we can compute a Gödel number of the sequence

$$
a, b, a, b, a, b, \ldots
$$

from $H_{a}$. We then obtain $c$ by the remark just before the theorem. This completes the proof of the theorem.

The remark before the theorem can now be extended as follows. Suppose that $\alpha$ is recursive in $F$ and that $\alpha(n) \in O$ for all $n$. Then there is a $b \in O$ such that $|\alpha(n)|$ $<|b|$ for all $n$. Moreover, $b$ may be computed from a Gödel number of $\alpha$ from $F$.

With these results, we can extend virtually all of the known results on the hyperarithmetical hierarchy which do not depend on the Souslin-Kleene theorem. For example, we may show that the ordinals $|a|$ for $a \in O$ are just the ordinals of wellordered sets of numbers which are recursive in $F$. The proof is essentially the same 
as Markwald's proof [4] in the ordinary case, making use of the result just mentioned.

Added in proof. Results similar to the above have been obtained by Hinman in his thesis (University of California at Berkeley, 1966).

\section{REFERENCES}

1. S. C. Kleene, On the forms of the predicates in the theory of constructive ordinals. I, Amer. J. Math. 66 (1944), 41-58; ibid., II, Amer. J. Math. 77 (1955), 405-428.

2. - Hierarchies of number-theoretic predicates, Bull. Amer. Math. Soc. 61 (1955), 193-213.

3. - Recursive functionals and quantifiers of finite types. I, Trans. Amer. Math. Soc. 91 (1959), 1-52; ibid., II, Trans. Amer. Math. Soc. 108 (1963), 106-142.

4. W. Markwald, Zur Theorie der konstruktiven Wohlordnungen, Math. Ann. 127 (1954), 135-149.

5. W. Richter, Constructive transfinite number classes, Bull. Amer. Math. Soc. 73 (1967), 261-265.

6. J. R. Shoenfield, "The form of the negation of a predicate" in Recursive function theory, pp. 131-134, Proc. Sympos. Pure Math., Vol. 5, Amer. Math. Soc., Providence, R. I., 1963.

7. C. Spector, Recursive well-orderings, J. Symbolic Logic 20 (1955), 151-163.

DUKe UNIVERSITY,

Durham, North Carolina 